\title{
Fusiones horizontales de empresas y estrategias de diferenciación de producto*
}

Firms horizontal mergers and product differentiation strategies

José MÉndez NAYA**

\begin{abstract}
Resumen
Partiendo de un modelo sencillo de oligopolio con diferenciación de producto, en el que hay tres empresas, el objeto de este trabajo es el de complementar la literatura existente sobre las fusiones horizontales de empresas probando que la tradicional paradoja de las fusiones puede evitarse si, como consecuencia de la fusión, las empresas fusionadas incrementan la diferenciación existente entre sus productos. En este contexto, se prueba que la fusión puede ser beneficiosa, desde el punto de vista del bienestar agregado, al incrementar el excedente de los consumidores y el beneficio de las empresas, tanto fusionadas como no fusionadas.
\end{abstract}

Palabras clave: Fusiones horizontales, diferenciación de producto, bienestar.

Clasificación JEL: L00, L13, L20.

\begin{abstract}
Taking as a reference a simple oligopoly model with differentiated products, in which there are three firms, the purpose of this paper is to complement the existing literature on mergers by proving that the traditional merger paradox can be avoided by assuming that, after the merger, the merging firms increase the existing bilateral product differentiation. In this context, it is proved that a merger could be welfare enhancing by increasing both consumers surplus and merging and non merging firms profits.
\end{abstract}

Key words: Horizontal mergers, product differentiation, welfare.

JEL classification: LOO, L13, L20.

* El autor agradece los valiosos comentarios de dos evaluadores anónimos. Cualquier error que presente el trabajo es responsabilidad exclusiva del autor. También se agradece el apoyo financiero de la Consellería de Innovación e Industria de la Xunta de Galicia por medio del proyecto 10 PXIB 100189 PR.

** José Méndez Naya; Facultad de Ciencias Económicas y Empresariales, Universidad de A Coruña, Campus de Elviña s/n, 15071 A Coruña, Spain. Teléfono: 981-167000, Fax: 981-167070, E-mail: jmn@udc.es.

Received: December, 2015. Accepted: July, 2016. 


\section{INTRODUCCIÓN}

La literatura existente sobre las fusiones horizontales de empresas establece que, como consecuencia de una fusión, tanto las empresas fusionadas como las no fusionadas pueden cambiar sus estrategias de comportamiento óptimo. Dicho cambio de estrategias afecta a todas las variables relevantes del análisis: beneficios de las empresas, tanto fusionadas como no fusionadas, excedente de los consumidores y bienestar agregado.

Partiendo de un modelo de oligopolio en el que las empresas producen un producto homogéneo y compiten a la Cournot, asumiendo que la demanda y los costes de producción son lineales y simétricos, el trabajo seminal de Salant et al. (1983) prueba el tradicional resultado conocido como la paradoja de las fusiones, (Pepall, et al., 1999). Dicha paradoja establece que una fusión resultará perjudicial para las empresas fusionadas excepto que se fusionen al menos el $80 \%$ de las empresas del mercado y al mismo tiempo, dicha fusión, será beneficiosa para las empresas no fusionadas.

Sin embargo, existe una importante contradicción entre el anterior resultado teórico y la evidencia empírica. De hecho, en la práctica, se ha producido un incremento exponencial en los procesos de fusión y adquisición de empresas en las últimas décadas y esta tendencia se ha producido en la mayor parte de los países industrializados y ha afectado a la mayoría de las industrias y sectores productivos. En este sentido, puede afirmarse que en las últimas seis décadas los procesos de fusión y adquisición de empresas constituyen un aspecto importante de las estrategias de crecimiento empresarial en numerosos sectores productivos y de servicios. Para una revisión retrospectiva de la evolución empírica de las fusiones como estrategia empresarial véase por ejemplo Scherer (2006).

Tomando como referencia el trabajo seminal de Salant et al. (1983), muchos autores han tratado de reconciliar la teoría con la evidencia empírica cambiando una o varias de las hipótesis del modelo original. Por ejemplo, una fusión puede ser beneficiosa si: las empresas participantes son suficientemente asimétricas, las funciones de costes y demanda son suficientemente convexas, cambia la estructura del juego o existen importantes ganancias de eficiencia entre las empresas fusionadas como consecuencia de la fusión. Para un análisis detallado de estos aspectos véase entre otros, Farrell y Shapiro (1990), Ding et al. (2013), Perry y Porter (1985) y Deneckere y Davidson (1985).

En concreto, Farrell y Shapiro (1990) y Perry y Porter (1985), establecen condiciones bajo las cuales una fusión que es beneficiosa para las empresas fusionadas, supone, al mismo tiempo, un incremento en el bienestar agregado del país. En la misma línea, cabe destacar los trabajos de Huck et al. (2004) y de Creane y Davidson (2004), que justifican que una fusión puede ser beneficiosa para las empresas fusionadas y suponer un incremento en el nivel de bienestar agregado debido a que las empresas fusionadas gozan de ciertas ventajas informativas que no están al alcance de las empresas no fusionadas.

Por otro lado, a pesar de que la literatura tradicional sobre las fusiones se ha centrado en el análisis de los efectos de las mismas asumiendo producto homogéneo, es evidente que la mayor parte de las fusiones se desarrollan en 
sectores en los que existe diferenciación de producto y, en la práctica, el grado de diferenciación de producto existente es una variable clave a la hora de analizar los efectos derivados de una fusión horizontal de empresas. Para un análisis empírico de los efectos de las fusiones en contextos de diferenciación de producto, véanse Werden y Froeb (1994), Ivaldi y Verboven (2005), Liu (2014) y Nevo (2000) entre otros.

A pesar de su evidente relevancia empírica, son pocos los desarrollos teóricos que tratan de analizar los efectos derivados de una fusión en un contexto de diferenciación de productos. Dos excepciones a este planteamiento son los trabajos de Kao y Menezes (2010) y Escrihuela-Villar (2011). En su artículo Kao y Menezes (2010), basándose en el modelo de Singh y Vives (1984), prueban que una fusión puede suponer un incremento en el bienestar agregado si las empresas compiten en cantidades y los productos son sustitutivos cercanos. Por su parte Escrihuela-Villar (2011), demuestra que una fusión horizontal de empresas puede ser beneficiosa si como consecuencia de la fusión aumenta el grado de diferenciación de producto existente en el mercado.

El objetivo de este artículo es el de complementar la literatura existente sobre las fusiones horizontales de empresas al justificar que la paradoja de las fusiones puede evitarse, incluso bajo las hipótesis básicas del modelo de Salant et al. (1983), asumiendo que las empresas fusionadas disponen de ciertas estrategias que, en general, no pueden llevar a cabo las empresas no fusionadas (Huck et al., 2004, y Creane y Davidson, 2004). En concreto, se asume que las empresas fusionadas pueden utilizar estratégicamente el grado de diferenciación existente entre sus productos. En este contexto, se prueba que, para ciertos grados de diferenciación de producto, la fusión será beneficiosa para las empresas fusionadas y perjudicial para las empresas no fusionadas evitando, de este modo, la tradicional paradoja de las fusiones. Además, se justifica que si, como consecuencia de una fusión, las empresas fusionadas incrementan suficientemente el grado de diferenciación existente entre sus productos, dicha fusión supone un incremento tanto en el bienestar agregado como en el excedente de los consumidores.

Estos resultados, que reconcilian la teoría con la evidencia empírica al justificar los numerosos procesos de fusión y adquisición de empresas que se producen en sectores en los que existe diferenciación de producto, tienen importantes implicaciones en materia de política de competencia al remarcar que el grado de diferenciación de producto existente, tras la fusión, puede ser un factor clave a la hora de evaluar los efectos derivados de la misma. En concreto, se prueba que una fusión puede ser sostenible para las empresas fusionadas y beneficiosa en términos de bienestar agregado si las empresas fusionadas, como consecuencia de la fusión, incrementan suficientemente el grado de diferenciación existente entre sus productos.

El trabajo se estructura como sigue: en la sección II se presenta el modelo de base. En la sección III la determinación del equilibrio de mercado antes y después de la fusión. En la sección IV se realiza un análisis comparativo de los resultados para las empresas, tanto fusionadas como no fusionadas, consumidores y bienestar agregado. Finalmente en la sección V se presentan las principales conclusiones. 


\section{EL MODELO}

Tal y como se ha indicado, el análisis se desarrolla tomando como referencia un mercado de oligopolio en el que hay 3 empresas simétricas que producen productos diferenciados y que compiten a la Cournot.

Se asume, al igual que Singh y Vives (1984), que la función de utilidad de los consumidores es cuadrática, estrictamente cóncava y simétrica y está dada por:

$$
U\left(x_{1}, x_{2}, x_{3}\right)=x_{1}+x_{2}+x_{3}-\frac{1}{2}\left(x_{1}^{2}+x_{2}^{2}+x_{3}^{2}+2 b\left(x_{1} x_{2}+x_{1} x_{3}+x_{2} x_{3}\right)\right)
$$

En donde, $x_{1}, x_{2}$ y $x_{3}$, representan las cantidades producidas por cada una de las tres empresa y el parámetro $0<b<1$ indica el grado de sustitución existente entre los productos. Si el valor de $b$ es próximo a la unidad significa que los productos son sustitutivos cercanos, poco diferenciados, y si su valor está próximo a cero significa que los productos tienden a ser independientes y por tanto muy diferenciados.

En este caso, el excedente de los consumidores, que es la diferencia entre la utilidad derivada del consumo y el gasto que supone el bien, está dado por:

$$
E C=U\left(x_{1}, x_{2}, x_{3}\right)-\sum_{i=1}^{3} p_{i} x_{i}
$$

La maximización de utilidad por parte de los consumidores da lugar a funciones inversas de demanda lineales, que vienen dadas por las siguientes expresiones:

$$
\begin{aligned}
& p_{1}=1-x_{1}-b x_{2}-b x_{3} \\
& p_{2}=1-x_{2}-b x_{1}-b x_{3} \\
& p_{3}=1-x_{3}-b x_{2}-b x_{1}
\end{aligned}
$$

En este caso, por la hipótesis de simetría, las funciones de costes de las tres empresas son idénticas y, para simplificar el análisis, se asume que no hay costes fijos de producción y los costes marginales son constantes e iguales a cero, con lo que la función de beneficios de cada una de las tres empresas estará dada por:

$$
\pi_{i}=p_{i} x_{i}, i=1,2,3
$$

El bienestar agregado del país, que está dado por la suma del excedente de los consumidores (EC) y el beneficio de las empresas, puede ser representado como sigue:

$$
W=E C+\sum_{i=1}^{3} \pi_{i}
$$




\section{EQUILIBRIO DE MERCADO ANTES Y DESPUÉS DE LA FUSIÓN}

En esta sección se determinará el equilibrio de mercado antes y después de la fusión de dos de las tres empresas.

\subsection{Determinación del equilibrio de mercado antes de la fusión}

A continuación, tal y como se ha indicado se procederá a la determinación del equilibrio de mercado previo al proceso de fusión.

Tal y como se ha indicado, se asume que las empresas compiten a la Cournot, es decir, cada empresa utiliza su estrategia de output tratando de maximizar su función de beneficio individual. La resolución del sistema de ecuaciones, resultante del anterior proceso, nos permite determinar los valores de output de equilibrio para las tres empresas que están dados por:

$$
x_{1}=x_{2}=x_{3}=\frac{1}{2(1+b)}
$$

Sustituyendo, pueden determinarse los valores de equilibrio correspondientes a los beneficios de las empresas, excedente de los consumidores y bienestar agregado que están dados respectivamente por las siguientes expresiones:

$$
\begin{aligned}
& \pi_{1}=\pi_{2}=\pi_{3}=\frac{1}{4(1+b)^{2}} \\
& E C=\frac{3(1+2 b)}{8(1+b)^{2}} \\
& W=\frac{3(3+2 b)}{8(1+b)^{2}}
\end{aligned}
$$

\subsection{Equilibrio de mercado después de la fusión}

De forma similar se procede a determinar el equilibrio de mercado después de la fusión, en este caso, tal y como se ha indicado, asumimos que las empresas fusionadas tienen la posibilidad de ajustar el grado de diferenciación existente entre sus productos y que dicha diferenciación no supone un coste adicional para las mismas ${ }^{1}$.

En concreto, suponiendo que se fusionan las empresas 1 y 2, las funciones inversas de demanda pueden escribirse como sigue:

$$
\begin{aligned}
& p_{1}=1-x_{1}-c x_{2}-b x_{3} \\
& p_{2}=1-x_{2}-c x_{1}-b x_{3} \\
& p_{3}=1-x_{3}-b x_{2}-b x_{1}
\end{aligned}
$$

1 La introducción de costes de diferenciación en el análisis no afectaría cualitativamente a los resultados, excepto que dichos costes fuesen muy elevados. 
En este caso, $0<c<1$ nos representa el grado de diferenciación existente entre los productos producidos por las empresas fusionadas. Si $c=b$, significa que las empresas fusionadas no varían el grado de diferenciación de los productos como consecuencia de la fusión. Por otro lado, si $c<b$ las empresas fusionadas deciden diferenciar en mayor medida los productos que producen respecto a la diferenciación existente en el mercado antes de la fusión y lo contrario ocurre si $c>b$.

Dado que estamos asumiendo una fusión entre las empresas 1 y 2, cada una de dichas empresas decidirá su estrategia de output con la finalidad de maximizar los beneficios agregados de ambas.

Procediendo como en la situación previa a la fusión, podemos determinar los valores de output de equilibrio para las tres empresas que están dados por:

$$
\begin{aligned}
& x_{1}^{F}=x_{2}^{F}=\frac{2-b}{2\left(2+2 c-b^{2}\right)} \\
& x_{3}^{F}=\frac{1+c-b}{2+2 c-b^{2}}
\end{aligned}
$$

En donde el superíndice ${ }^{\mathrm{F}}$ hace referencia a la situación posterior a la fusión. Dados los anteriores valores de output, se puede determinar el equilibrio de mercado que viene dado por las siguientes expresiones:

$$
\begin{aligned}
& \pi_{1}^{F}=\pi_{2}^{F}=\frac{(b-2)^{2}(c+1)}{4 b^{4}-16(1+c)\left(b^{2}-1-c\right)} \\
& \pi_{3}^{F}=\frac{(1+c-b)^{2}}{\left(2+2 c-b^{2}\right)^{2}} \\
& C S^{F}=\frac{b^{2}(6 b-5 c-17)+2\left(c^{2}+4 b(1+c)+3\right)}{4\left(2+2 c-b^{2}\right)^{2}} \\
& W^{F}=\frac{b^{2}(-4 b+c+3)+16 b(1+c)-6\left(c^{2}+4 c+3\right)}{4\left(2+2 c-b^{2}\right)^{2}}
\end{aligned}
$$

\section{AnÁlisis COMPARATIVo}

El objetivo de esta parte es el de analizar los efectos de la fusión sobre las variables relevantes del análisis y cómo influye, sobre los resultados, el grado de diferenciación establecido por las empresas fusionadas sobre sus productos.

Para desarrollar el análisis, se comparan los valores de los beneficios, tanto de las empresas fusionadas como no fusionadas, excedente de los consumidores y bienestar antes y después de la fusión.

Asumiendo que las empresas fusionadas no cambian el grado de diferenciación existente entre sus productos, es decir $\mathrm{c}=\mathrm{b}$, es fácil comprobar que la fusión, a pesar de ser beneficiosa para las empresas fusionadas si el grado de diferenciación existente es suficientemente grande $(b<0.55)$ y de ser siempre beneficiosa para la empresa no fusionada, resultará siempre perjudicial para los 
consumidores y además supondrá una reducción en el bienestar agregado de la economía. Estos resultados son consistentes con los obtenidos por Salant et al. (1983) para el caso de producto homogéneo.

A continuación, se analizara en qué medida los anteriores resultados se ven afectados asumiendo que las empresa fusionadas pueden ajustar el grado de diferenciación existente entre sus propios productos.

\subsection{Efectos de la fusión sobre los beneficios de las empresas fusionadas}

La comparación de los beneficios correspondientes a las empresas fusionadas antes y después de llevar a cabo la fusión nos permite enunciar el siguiente resultado:

\section{Resultado 1}

Una fusión entre dos empresas será sostenible siempre que, como consecuencia de la fusión, las empresas fusionadas incrementen suficientemente el grado de diferenciación existente entre sus productos.

\section{Prueba}

En este caso, puede razonarse del siguiente modo:

$$
\text { Sea } c^{1}=\frac{1}{8}\left[(b-1)\left(b^{3}-b^{2}+4-\sqrt{b^{4}-2 b^{3}+5 b^{2}+4 b+4}(b+1)\right)(b-2)\right],
$$

se verifica que:

$\pi_{i}^{F}=\pi_{i}>0 \Leftrightarrow c<c^{1}, \forall i=1,2$. Con lo que queda demostrado.

La relación existente entre $c^{1}$ y $b$ puede visualizarse con la ayuda de la Gráfica 1:

\section{GRÁFICA 1}

EFECTOS DE LA FUSIÓN SOBRE EL BIENESTAR AGREGADO Y SUS COMPONENTES

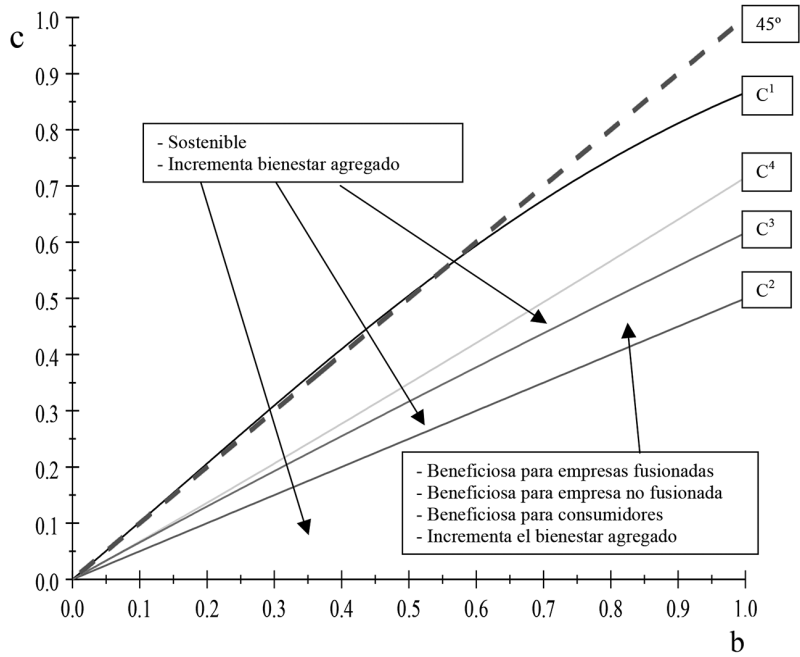


La función $c^{1}$ nos da las combinaciones de $b$ y $c$ que hacen que las empresas fusionadas sean indiferentes entre fusionarse y no fusionarse. Por encima de la función $c^{1}$ las empresas que se fusionan se ven perjudicadas como consecuencia de la fusión y por debajo de $c^{1}$ los beneficios de las empresas fusionadas se ven incrementados como consecuencia de la fusión y por tanto, dicha fusión, es sostenible. Puede observarse en la gráfica que si las empresas fusionadas no varían el grado de diferenciación existente entre sus productos, $b=c$, la fusión será sostenible, tal y como se ha indicado, si $b<0.5$.

El anterior resultado nos indica que la fusión entre dos empresas será sostenible si para un cierto grado de diferenciación existente en el mercado antes de la fusión, las empresas fusionadas incrementan suficientemente el grado de diferenciación entre sus productos.

Este resultado se debe a que al aumentar la diferenciación existente entre sus productos, las empresas fusionadas reducen el efecto de canibalización entre los mismos, de este modo, se puede compensar el tradicional efecto negativo sobre las empresas fusionadas, como consecuencia de la fusión, que se deriva de la reducción de las ventas agregadas de las empresas fusionadas y del incremento de las ventas de la tercera empresa.

\subsection{Efectos de la fusión sobre la empresa no fusionada}

El análisis puede desarrollarse, de forma similar al caso anterior, comparando los beneficios de la tercera empresa antes y después de la fusión, lo que nos permite enunciar el siguiente resultado:

\section{Resultado 2}

La empresa no fusionada se beneficiará de la fusión siempre que, como consecuencia de la fusión, las empresas fusionadas no incrementen excesivamente el grado de diferenciación existente entre sus productos.

Prueba:

Sea $c^{2}=\frac{1}{2} b$, en este caso, se verifica que: $\pi_{3}^{M}-\pi_{3}>0 \Leftrightarrow c>c^{2}$, lo que prueba el resultado.

De nuevo, podemos ilustrar este resultado con la ayuda de la Gráfica 1, en donde la función $c^{2}$ nos da las combinaciones de $b$ y $c$ que hacen que la fusión no le afecte al beneficio de la empresa no fusionada. Por encima de la función $c^{2}$ las empresas no fusionadas ven incrementados sus beneficios como consecuencia de la fusión y por debajo de $c^{2}$ ocurre lo contrario.

En este caso, se producen dos efectos de signo opuesto sobre el nivel de output y sobre los beneficios de la empresa no fusionada: por un lado un efecto positivo derivado de la cooperación entre las dos empresas fusionadas, que reducen su nivel de producción lo que supone un aumento en el output de la empresa no fusionada, y por otro lado, el efecto negativo derivado del incremento en la diferenciación de producto, lo que resta cuota de mercado a la empresa no fusionada. 


\subsection{Efectos sobre el excedente de los consumidores y sobre el bienestar agregado}

Este es un aspecto muy importante desde el punto de vista de la política de competencia ya que tradicionalmente las fusiones de empresas son vistas como algo negativo para los consumidores, e incluso para el bienestar agregado, en la medida en que suponen una concentración de empresas con la consiguiente reducción de la competencia. El objetivo de esta parte es el de analizar en qué medida los anteriores resultados se ven afectados por el hecho de que las empresas fusionadas puedan variar el grado de diferenciación de sus productos como consecuencia de la fusión.

La comparación de los excedentes de los consumidores y niveles de bienestar agregado antes y después de la fusión nos permite enunciar el siguiente resultado:

\section{Resultado 3}

La fusión supondrá un incremento tanto en el excedente de los consumidores como en el bienestar agregado siempre que el grado de diferenciación de producto de las empresas fusionadas aumente suficientemente como consecuencia de la fusión.

\section{Prueba}

En lo que se refiere al excedente de los consumidores puede argumentarse como sigue:

Sea $\quad c^{3}=\frac{6 b^{5}-13 b^{4}-16 b^{3}+22 b^{2}+16 b}{2\left(5 b^{4}-10 b^{3}-17 b^{2}+16 b+12\right)}, \quad$ puede probarse que: $C S^{M}-C S>0 \Leftrightarrow c<c^{3}$.

Porotrolado, respectoal bienestaragregado, sea $c^{4}=\frac{2 b^{5}+b^{4}-12 b^{3}+2 b^{2}+16 b}{2\left(b^{4}+6 b^{3}-9 b^{2}-8 b+12\right)}$, en este caso, se verifica que: $W^{M}-W>0 \Leftrightarrow c<c^{4}$, con lo que queda demostrado.

Una vez más, la Gráfica 1 nos permite visualizar este resultado, en la misma las funciones $c^{3}$ y $c^{4}$ representan, respectivamente, las combinaciones de parámetros que hacen que el excedente de los consumidores y el bienestar agregado no se vean afectados por la fusión. Por encima de las funciones $c^{3}$ y $c^{4}$ el excedente de los consumidores y el bienestar, respectivamente, se ven reducidos y lo contrario ocurre por debajo de dichas funciones.

En este caso los consumidores se ven beneficiados directamente por el aumento en el grado de diferenciación de los productos de las empresas fusionadas e indirectamente porque, al ser mayor dicho grado de diferenciación, aumentan las ventas totales del mercado.

Por otro lado, al aumentar el grado de diferenciación, a pesar de que la empresa no fusionada ve reducidos sus beneficios como consecuencia de la 
fusión, el incremento tanto en los beneficios de las empresas fusionadas como en el excedente de los consumidores, supone un aumento en el bienestar agregado del país.

\subsection{Efectos globales derivados de la fusión}

En esta parte se procede a un análisis conjunto de los efectos derivados de la fusión sobre todas las variables relevantes en función del grado de diferenciación existente entre los productos de las empresas fusionadas después de la fusión respecto a la diferenciación de producto existente antes de la fusión.

Del análisis anterior se derivan los siguientes resultados:

\section{Resultado 4}

Para ciertos grados de diferenciación entre los productos de las empresas fusionadas, una fusión siempre será sostenible y beneficiosa desde el punto de vista del bienestar agregado.

Prueba

En este caso, se verifica que $c^{1}>c^{4}>c^{3}>c^{2}, \forall 0<b<1$, y dado que la fusión es sostenible siempre que $c<c^{1}$ e incrementa el nivel de bienestar si $c<c^{4}$, se verifica que será sostenible y al mismo tiempo incrementa al bienestar agregado siempre que $c<c^{4}$.

\section{Resultado 5}

Dada una cierta diferenciación de producto en el mercado antes de la fusión siempre existe un grado de diferenciación entre los productos de la empresa fusionada tal que tanto el bienestar agregado como todos sus componentes aumentan como consecuencia de la fusión.

Prueba

Dado que, $c^{1}>c^{4}>c^{3}>c^{2}, \forall 0<b<1$, y al mismo tiempo, se verifica que la fusión: es sostenible siempre que $c<c^{1}$, incrementa el nivel de bienestar si $c<c^{4}$ y es beneficiosa para las empresas no fusionadas si $c>c^{2}$. Entonces la fusión supondrá un incremento del bienestar agregado y todas sus componentes si $c^{3}>c>c^{2}$.

La Gráfica 1 muestra las zonas, delimitadas por las funciones representadas, en las que se verifican los resultados 4 y 5 .

Tal y como se ha indicado, en el área comprendida entre $c^{1}$ y $c^{4}$ la fusión es beneficiosa para las empresas fusionadas y al mismo tiempo incrementa el bienestar social. Por otro lado en el área comprendida entre $c^{3} y c^{2}$, la unión no solo es sostenible y beneficiosa en términos de bienestar agregado sino que también es beneficiosa tanto para los consumidores como para la empresa no fusionada. 


\section{Conclusiones}

Partiendo de un modelo sencillo de oligopolio en el que las empresas producen productos diferenciados, en este trabajo se trata de complementar la literatura existente sobre la sostenibilidad de las fusiones y sus efectos tanto sobre el bienestar agregado como sobre cada una de sus componentes.

El objetivo del trabajo es del de reconciliar la teoría con la evidencia empírica al probar que la tradicional paradoja de las fusiones, que se produce bajo las hipótesis básicas del modelo de Salant et al. (1983), no tiene porque producirse si se cambia alguna de las hipótesis de dicho modelo. Específicamente, de forma similar a Huck et al. (2004) y de Creane y Davidson (2004), se asume que las empresas fusionadas disponen de ciertas posibilidades, en lo que se refiere a la reorganización interna de su producción, que en general no están a disposición de las empresas no fusionadas. Concretamente, se asume que después de la fusión, las empresas fusionadas pueden ajustar el grado de diferenciación existente entre los productos que producen.

En este contexto se justifica que el grado de diferenciación de producto existente tras la fusión es una variable clave a la hora de analizar tanto la sostenibilidad de la misma como sus efectos sobre el bienestar. En concreto, se prueba que si las empresas fusionadas incrementan suficientemente el grado de diferenciación entre sus productos tras la fusión la fusión, aparte de ser beneficiosa para las empresas fusionadas, supondrá un incremento en del bienestar agregado del país.

El anterior resultado tiene importantes implicaciones para la política de competencia dado que, en general, se considera que los procesos de concentración empresarial son negativos desde el punto de vista del bienestar agregado ya que suponen una reducción en la competencia de mercado. Sin embargo, esta reducción en la competencia de mercado podría verse compensada si, tal y como se ha indicado, las empresas fusionadas incrementan suficientemente el grado de diferenciación entre sus productos de tal modo que el efecto neto sobre el bienestar agregado puede resultar positivo.

\section{Bibliografía}

Creane, A. y Davidson, C. (2004). "Multidivisional Firms, Internal Competition, and the Merger Paradox". Canadian Journal of Economics, Vol. 37, 951-77.

Deneckere, R. y Davidson, C. (1985). "Incentives to Form Coalitions with Bertrand Competition". The Rand Journal of Economics, vol. 16 (4), 473-486.

Ding,W., Fan, C. y Wolfstetter, E. (2013)". Horizontal Mergers with Synergies: Cash-vs. Profit-Shareauctions". International Journal of Industrial Organization, vol. 31, 382-391.

Escrihuela-Villar, M. (2011). "On Welfare Effects of Horizontal Mergers with Product Differentiation". Journal or Economics Finance and Administrative Science, vol. 16 (30), 8,12.

Farrell, J. y Shapiro, C. (1990). "Horizontal Mergers: An Equilibrium Analysis". American Economic Review, vol. 80, 107-126.

Huck, S., Konrad, K. A. y Müller, W. (2004). "Profitable Horizontal Mergers without Costs Advantages: The role of Internal Organization, Information and Market Structure". Economica, Vol. 71, 575-587. 
Ivaldi, M. y Verboven, F. (2005). "Quantifying the effects from horizontal Mergers in European Competition Policy". International Journal of Industrial Organization, vol. 23 (9-10), 669-691.

Kao, T. y Menezes, F. (2010). "Welfare-Enhancing Mergers Under Product Differentiation". The Manchester School, vol. 78 (4), 290-301.

Liu, Y. (2014). "Should Further Merger Be Allowed? Product Differentiation and Merger in the External Audit Market". Applied Economics, vol. 46(7), 74-749.

Nevo, A. (2000). "Mergers with Differentiated Products: the Case of the Case of the Ready-To-Eat Cereal Industry". The RAND Journal of Economics, vol. 31, 395-421.

Pepall, L., Richards, D. J. y Norman, G. (1999). Industrial Organization: Contemporary Theory and Practice, South-Western College Publishing.

Perry, M. K. y Porter, R. H. (1985). "Oligopoly and the incentives for horizontal mergers”. American Economic Review, vol. 75, 219-227.

Salant, S., Switzer, S. y Reynolds, R. J. (1983). "Loses from Horizontal Merger: the Effects of an Exogenous Change in Industry Structure on CournotNash Equilibrium". Quarterly Journal of Economics, vol. 48, 185-200.

Scherer, R. M. (2006). "A New Retrospective on Mergers", Review of Industrial Organization, vol. 28, pp. 327-341.

Singh, N. y Vives, X. (1984). "Price and Quantity Competition in a Differentiated Duopoly", Rand Journal of Economics, 15(4), pp. 546-554.

Werden, G. y Froeb, L. (1994). "The Effects of Mergers in Differentiated Products Industries: Logit Demand and Merger Policy". International Journal of Law, Economics, and Organization, vol. 10(2), 407-426. 